\title{
Annihilation Mechanisms
}

\author{
Dirk J. Pons ${ }^{1}$, Arion D. Pons ${ }^{1} \&$ Aiden J. Pons ${ }^{2}$ \\ ${ }^{1}$ Department of Mechanical Engineering, University of Canterbury, Christchurch, New Zealand \\ ${ }^{2}$ Rangiora New Life School, Rangiora, New Zealand \\ Correspondence: Dirk J. Pons, Department of Mechanical Engineering, University of Canterbury, Private Bag \\ 4800, Christchurch 8020, New Zealand. E-mail: dirk.pons@canterbury.ac.nz
}

Received: December 24, 2013 Accepted: January 8, 2014 Online Published: February 20, 2014

doi:10.5539/apr.v6n2p28 URL: http://dx.doi.org/10.5539/apr.v6n2p28

\begin{abstract}
This paper develops an ontologically rich explanation of the inner mechanics of the annihilation process, starting from a non-local hidden-variable (NLHV) design. This explains the process in terms of the handedness of matter and antimatter, the interaction of the electron and antielectron as they approach, the collapse of their discrete force structures and their reformation into photon structures. The process is more one of remanufacture than destruction. The resulting Cordus theory successfully explains para- and ortho-positronium annihilation. It explains the different photons output, the relative difference in lifetimes, and why Bhabha scattering sometimes happens instead. The theory exposes a deeper common mechanism for annihilation, pair-creation, and bonding.
\end{abstract}

Keywords: annihilation, positronium, parapositronium, orthopositronium, pair-creation, positron

\section{Introduction}

How do matter and antimatter annihilate? Why does it happen at all? These ontological questions are not answered by conventional physics. This paper offers an explanation of the mechanics of the annihilation process. It uses a design from the non-local hidden-variable (NLHV) sector, specifically the Cordus theory. This theory has previously been used to reconceptualise wave-particle duality, explain entanglement ( D. J. Pons, A. D. Pons, A. M. Pons, \& A. J. Pons, 2012), identify the mechanics of the strong force (D. J. Pons, A. D. Pons, \& A. J. Pons, 2013c), and address several cosmological phenomena (D. J. Pons, A. D. Pons, \& A. J. Pons, 2013a; D. J. Pons \& A. D. Pons, 2013). The present paper complements these concepts by developing an explanation for how the structures of the particles interact and bring about the annihilation process under a NLHV scenario.

\section{Existing Theories of Annihilation}

The process of annihilation can be represented by Feynman diagrams, albeit at a high level of abstraction. However these diagrams do not represent the underlying mechanisms at the deeper level, nor all the intermediate processes. In the latter regard the diagrams are consistent with empirical observed tracks, where certain intermediates are not detected until a transformation to another particle occurs. The diagrams encapsulate the idea that these unobservable structures are 'virtual' particles. Thus virtual bosons are identified as part of the deconstruction process, and in the Standard Model even the photon is repurposed as a virtual photon for the electromagnetic effect.

Existing approaches to annihilation are primarily the fitting of mathematical models to empirical observations. This is a complex problem because of the diversity of outputs. Typical focus areas are on the production channels for various combinations of outcomes (Bardin \& Riemann, 1996), the conditions under which they arise (Honda et al., 2007), and the output characteristics (Kurihara, Fujimoto, Munehisa, \& Shimizu, 1996; Lampe \& Kramer, 1983) including jet width (Adachi et al., 1999) and energies involved (Abak, 1976). The practical measurement of annihilation data typically involves smashing particles together in colliders, and this introduces additional complexity into the process. For a start, the input particles are not always pure electrons and antielectrons. Instead they may be proton vs. proton. Secondly, the input particles have considerable kinetic energy. Thus experiments in high energy physics may produce complex showers of various short-lived particles and antiparticles that further decay into other outputs (Renard, 1976).

Mathematical models have been constructed to account for production rates under various types of annihilation, e.g. for e+e- into photons (Abak, 1976; Lampe \& Kramer, 1983; Kurihara et al., 1996), leptons or muons 
(Lyuboshitz, 2008, 2009; Gronau, Smith, Walsh, Wolfram, \& Yang, 1977). There is also work on hadrons (Field \& Wolfram, 1983; Kazama \& York-Peng, 1979; Pei, 1996), positronium output states (Frolov, Kryuchkov, \& Smith, 1995), and hydrogen-antihydrogen annihilation (Jonsell, Saenz, Froelich, Zygelman, \& Dalgarno, 2001). These approaches are called descriptions of the process, but they do not describe as much as fit mathematical models and coefficients to the empirical data (Jean et al., 2006; Renard, 1976; Chao, Meng, \& Pan, 1986). In general these require tuning to fit empirical observations. The models may usefully be applied in the inverse direction, by examining astronomical emissions, and inferring the environmental conditions at the source (Jean et al., 2006). Overall, the resulting aggregation of mathematical methods has empowered the quantum chromodynamics (QCD) model with good fit to empirical data (Brandelik et al., 1980; Field \& Wolfram, 1983). The ultimate hope with this particular approach is to explain the mechanisms for hadron production (Pei, 1996), though this is a goal not yet achieved.

In summary, the mathematical models provide good fit to the annihilation data, but the descriptive understanding of the underlying mechanism is lacking (Note 1). The production mechanisms themselves remain obscure, even if the outputs can be predicted and modelled accurately. It is this gap that the present paper targets, by providing a conceptual theory of the production processes for annihilation.

\section{Purpose and Methodology}

Purpose: The purpose of this paper is to explain the annihilation process from first principles, starting from a non-local hidden-variable (NLHV) design. This is worth attempting for the potential to better understand the processes underpinning mass-energy equivalence. The area under examination is the annihilation of an electron and anti-electron (positron), i.e. positronium. This is a gedanken experiment, and seeks to develop a new conceptual theory for the area under examination. This is a hidden variable approach, and is unlike quantum mechanics with its assumption that particles are merely zero-dimensional points. The starting position here is an acceptance of the premise of physical realism: that physical form exists and underpins the functional behaviour observed at the fundamental level.

Method: A systems engineering design methodology was used, whereby the requisite structure of the inner system is inferred from the observed or required external behaviour of the system. This is a conceptual methodology, and produces a qualitative explanation, as opposed to a mathematical formalism. This method seeks to infer, using creative design, the relationships of causality within a system as a whole. Here the specific system is positronium annihilation, and the problem is: given the known characteristics of the electron and antielectron for the NLHV solution under consideration, and accepting the empirical evidence for positronium annihilation, infer the mechanics that would be conceptually sufficient to explain the causality at this level, under the constraints that such inferred mechanics should be parsimonious (conceptually efficient) and logically integrated with other extant mechanisms within that NLHV theory (internal conceptual coherence). This involves creating various solutions (proposed mechanics for annihilation) and testing them in thought-experiments against known phenomena and empirical results, to select the fittest solution. The implications of those mechanics are determined for the wider theory (which covers other fundamental and cosmological phenomena using the same core NLHV design), and either the new or the existing mechanics revised as necessary. We represent this theory as a causal model, using the systems engineering modelling notation of integration definition zero (IDEF0) (FIPS, 1993). The IDEF0 model represents the proposed relationships of causality, and thus serves the same purpose as mathematical formalism does in conventional physics. (With IDEF0 the object types are inputs, controls, outputs, and mechanisms (ICOM) and are distinguished by placement relative to the box, with inputs always entering on the left, controls above, outputs on the right, and mechanisms below.)

Approach: The first activity in the systems design process was to design a NLHV solution for wave-particle duality (Pons et al., 2012). This was called the Cordus conjecture. It showed that a specific physical structure for particles is able to provide a logically consistent explanation to these effects. This structure has string-like attributes, hence cordus, and is called a particule to differentiate it from the zero-dimensional point-particle construct of quantum mechanics. The second activity was to find a workable design for the field structures. A logical necessity of such particules would be their emission of discrete forces in flux tubes (called hyperfine fibrils hence hyff). Design principles suggested that the emission would be in three orthogonal directions (hence hyff emission directions or HEDs). The theory then explains the aggregation of these as the electro-magneto-gravitational (EMG) fields, which are thus macroscopically smooth but fundamentally discrete. Within this theory the strong force is represented by a synchronous interactions between the discrete forces (Pons et al., 2013c). From this arose a qualitative explanation for the table of nuclides ( $\mathrm{H}$ to Ne) (D. J. Pons, A. D. Pons, \& A. J. Pons, 2013b). The field component of the Cordus theory yielded a novel theory for time as an emergent property of matter (Pons et al., 2013a), and an explanation for the cosmological horizon ( D. J. Pons \& A. D. 
Pons, 2013). Consequently the theory provides a conceptual unification between the fundamental scale where quantum mechanics usually operates, and the cosmological scale of general relativity. The third work-stream was to create a means to differentiate between matter and antimatter within this conceptual framework. The design that emerged as being sufficient and also consistent with the wider theory, was that fundamental difference between matter and antimatter is the hand of their discrete fields. Hand corresponds to the energisation sequence of three orthogonal discrete field elements. There are only two unique ways this can be done, which we term dexter and sinister for matter and antimatter respectively (D. J. Pons, A. D. Pons, \& A. J. Pons, 2013 (accepted)). The HED notation shows the ways that different particules energise their discrete fields, and permits identification of the charge (number of HEDs and direction of emission), and hand (energisation sequence). We find this to be a useful tool in expressing particule interactions.

The fourth activity, and the subject of this paper, was to determine the fundamental processes whereby the electron and antielectron annihilate. We sought the underlying mechanisms for mass-energy equivalence. A solution for this was found by extending the theory for discrete forces. Specifically, the design methodology applied to the synchronous interaction of discrete forces from opposite handed particules (hence matter-antimatter) resulted in a solution: a process is found whereby the discrete forces of massy particules would convert to those of the photon, i.e. mass conversion to energy.

The results are a set of process diagrams for the annihilation process. These are conceptual representations of the theory, as opposed to mathematical formalisms. In terms of the design methodology used here, these results correspond to a concept-design, which is a necessary preliminary artefact that would be further evaluated and eventually detailed further (e.g. in mathematical terms).

The annihilation solution presented here is logically consistent with all the other parts of the Cordus theory (see above), and was found not to require major revision of that theory to accommodate these developments, i.e. the results have internal validity. The results demonstrate that the NLHV design under consideration has the ability to provide a coherent physical explanation for the process of annihilation. Several basic principles become evident in this annihilation process, and we believe that the mechanisms are applicable to more complex particle combinations too. We close by demonstrating how the theory applies to ortho- and para-positronium.

\section{Results}

A brief summary is first given of the Cordus theory, since this is a foundational concept.

\subsection{Cordus Theory}

The Cordus theory postulates that all particles are really linear structures (hence cordus), and from their two ends emit three-dimensional discrete forces that travel down flux lines (Cordus: hyperfine fibril or hyff). This structure is called a particule. The two reactive ends are a small finite distance apart (span). A fibril joins the reactive ends and is a persistent and dynamic structure but does not interact with matter. It provides instantaneous connectivity and synchronicity between the two reactive ends, which are energised sequentially at the de Broglie frequency. The result is a non-local solution as the particule is affected by incoming discrete forces within the range of its reactive ends, as opposed to merely the fields at its nominal centre point. Thus locality fails but a principle of Wider Locality applies. For a fuller discussion see (Pons et al., 2013c) (Note 2).

\subsection{Structures of the Participant Particules: Photon, Electron, Antielectron}

The Cordus theory has specific predictions for the structure of each of the photon, electron, and antielectron particules. The requirements for the proton structures arose elsewhere, from considerations of wave-particle duality and entanglement (Pons et al., 2012), rather than for annihilation reasons. The electron and antielectron designs arose from consideration of the HED mechanics and handedness of matter-antimatter (Pons et al., 2013 (accepted)). Within this theory the HED arrangements determine the type of particule, and these differences are briefly summarised below since they are important in understanding the Cordus theory for annihilation.

\section{Photon structures}

Characteristics of the photon in the Cordus theory are:

- The reactive ends of the photon are energised together, whereas those of all other (matter) particules are energised reciprocally.

- The photon has a single discrete force at each end, which it extends and retracts, whereas other massy particules emit their discrete forces into a flux tube, let them go, and create new ones. Hence an evanescent field for the photon.

- The absolute direction of the discrete forces is the same at the two reactive ends of the photon, at any 
one moment in its energisation cycle. In contrast the discrete forces of massy particules are in the same relative direction (inwards or outwards) at their two ends.

- The result is an external structure of oscillating discrete forces. This is caused by close coupling between the external field and the fibril: the energy oscillates between the two. Thus the field changes sign, hence the observed reality that the electric field of the electromagnetic light wave reverses sign.

The photon structure is shown in Figure 1.

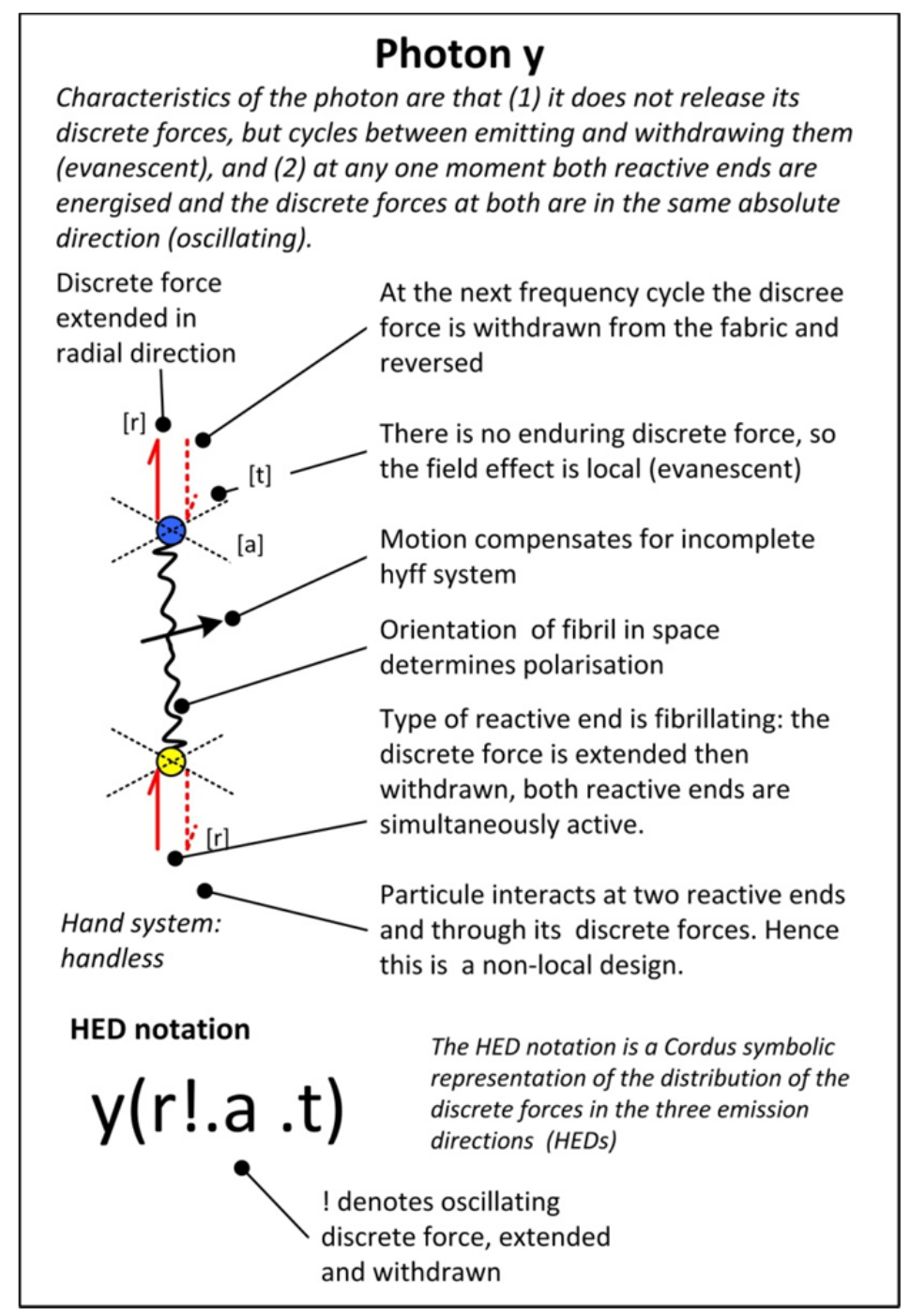

Figure 1. The Cordus theory for the photon structures

\section{Electron structures}

In this theory electric charge is carried at $1 / 3$ charge per discrete force, with the sign of the charge being determined by the direction of the discrete force element. The discrete forces are connected in a flux line that is emitted into the external environment. Each reactive end of the particule emits three such orthogonal hyff, at least in the near-field, see the electron in Figure 2. These directions are relative to the orientation of the span, and the velocity of the particule, and termed hyperfine-fibril emission directions (HEDs). The axes are named [r] radial outwards co-linear with the span, [a] and [t] perpendicular to the span and to each other. These are so-named for consistency with the nomenclature for the photon, but when applied to massy particules do not necessarily imply motion. 


\section{Electron e}

Characterised by one discrete force in each of the three directions. Therefore a highly stable structure. Together with anti-electron is manufactured from photons (pair production)

The discrete forces are released rather than retained as in the photon. Consequently there is an enduring hyff in each of the three directions, which creates a long-ranged force effect.

New discrete forces continue to be created and sent down the flux tube (hyff) at each frequency cycle

Fibril provides instantaneous communication between reactive ends

Type of reactive end: pulsatile. One reactive end energising and the other de-energising $\left(180^{\circ}\right.$ out of phase)

The HED notation is a Cordus symbolic representation of the distribution of the discrete forces in the three emission directions (HEDs)

\section{HED notation}

Three orthogonal axes $(r, a, t)$ for emission of discrete forces

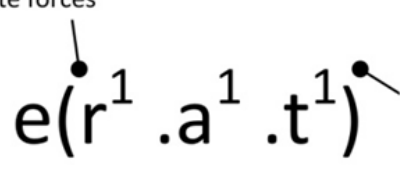

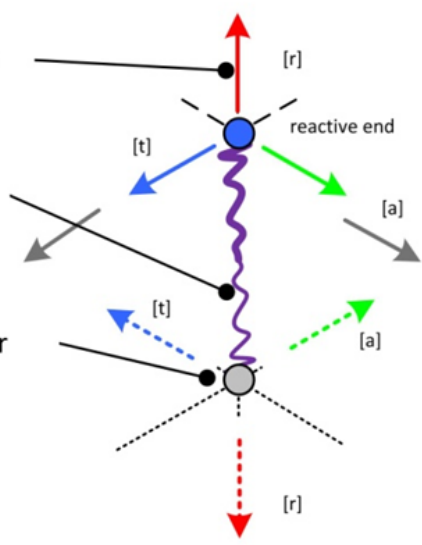

Dexter hand of energisation sequence for matter

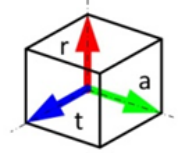

Each discrete force carries a $1 / 3$ electrical charge, with the sign representing the direction, so electron has overall -1 charge.

Figure 2. Internal and external structures of the electron according to the Cordus theory. It is proposed that the particule has three orthogonal discrete forces, energised in turn at each reactive end

The electron releases its discrete forces into the external environment, and then emits a new set at the next energisation cycle of that reactive end. The discrete force is emitted at one reactive end and then the other, in a pulsatile manner. Hence, when viewed at a coarser scale, such that the span is not evident and it looks like a point, the field system looks smooth.

\section{Contrasts}

In this theory the photon's discrete force is pushed into (or pulled from) a recruited volume of space and impeded in the process, hence the exponential reduction with range for the evanescent field. In contrast the electron discrete forces are propagated outwards as discrete forces that are not weakened with distance, and therefore their density is diluted across the surface of a growing sphere, hence a reduction with radius-squared for the EMG forces. Thus the theory differentiates between, and explains the origins of, the evanescent and electric fields. Polarisation of the photon is explained as orientation of the photon particle. Likewise spin of the electron is explained as the phase of energisation relative to another particule, and the two most interesting states are in phase (Cordus: 'cis-phasic') and opposite phase (Cordus: 'trans-phasic') (Pons et al., 2013c). These correspond to para and ortho spins states in quantum mechanics.

\section{Antielectron structure}

In this theory the matter-antimatter species are differentiated by the energisation sequence of the discrete forces in the three axes $[\mathrm{r}, \mathrm{a}, \mathrm{t}]$. This introduces a dexter-sinister (respectively) handedness to the discrete forces (Pons et al., 2013c). Antiparticules have opposite hand, i.e. the sequence of energisation of the discrete forces is 
spatially inverted (mirrored). Inversion of the hand also changes the direction of the discrete forces, hence the sign of the charge. This is consistent with the observation that the antielectron has positive charge. This theory uses the underscore to denote antimatter, rather than the overbar, since this is a new construct. The proposed Cordus structure theory for the antielectron, or positron, is shown in Figure 3.

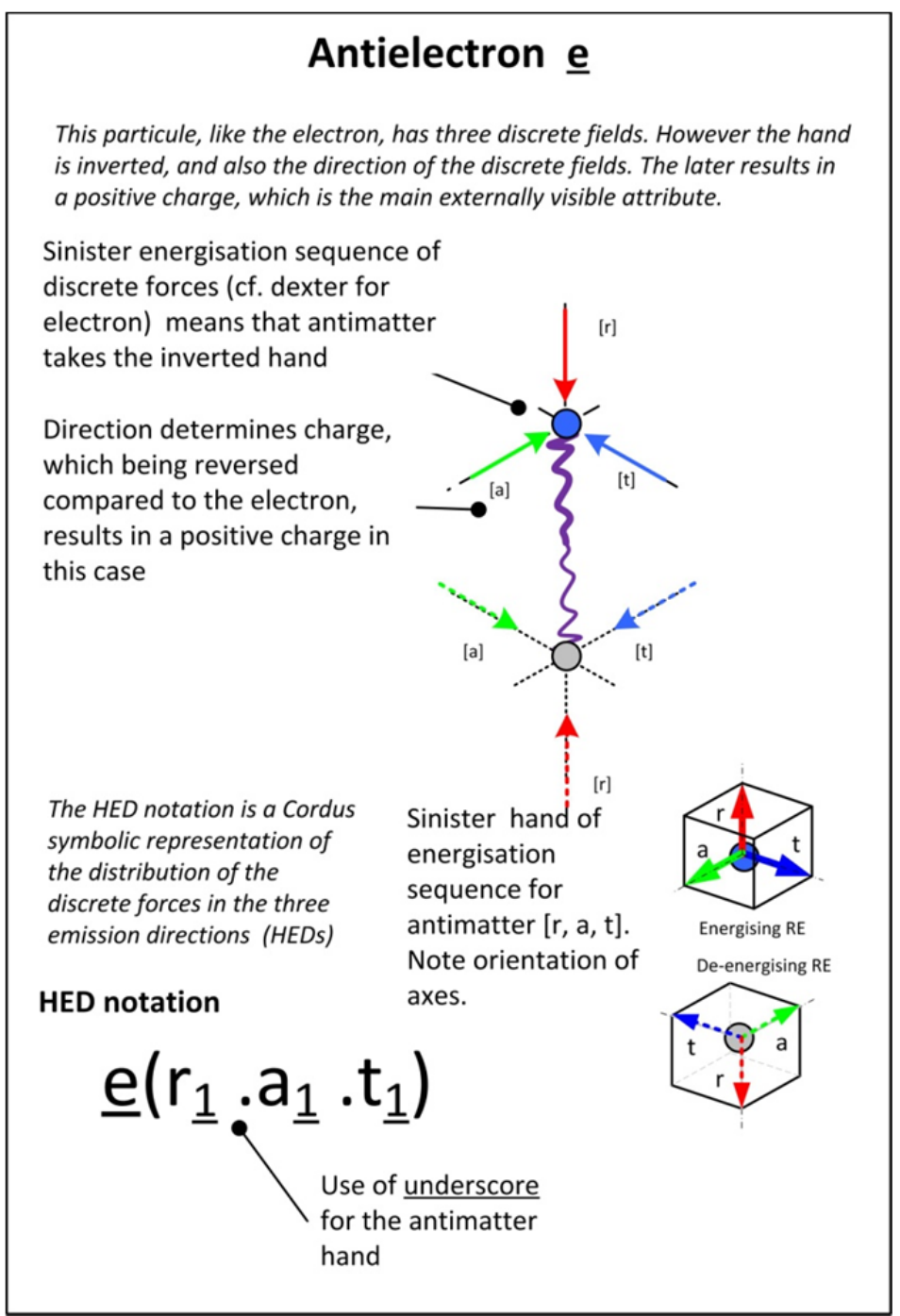

Figure 3. Cordus structure for the antielectron. It is proposed that the particule has three orthogonal discrete forces, energised in turn at each reactive end. The energisation sequence, hence hand, is opposite to that of the electron, and the direction of charge propagation is also reversed

\section{HED notation}

The HED notation is a shorthand symbolic representation of the discrete force arrangements for a particule, and includes the three axes $(\mathrm{r}, \mathrm{a}, \mathrm{t})$ and the number and direction of discrete forces in each. Charge corresponds to the direction of emission, negative outwards or positive inwards, and designated superscript and subscript respectively. The matter-antimatter handedness is designated by an underscore for the antiparticule.

All particules are uniquely distinguished by their discrete force emission, i.e. their HED signature. This is how all (say) electrons know how to behave: the only interaction the particule makes with the external environment is through its discrete fields.

The synchronicity between discrete force elements of neighbouring particules provides the strong force. Assembled massy particules compete spatially for emission directions, and may synchronise their emissions to access those spaces. Thus there is mutual negotiation in the near-field between interacting particules, based on 
shared geometric timing constraints. These particules interact by negotiating complementary HEDs and synchronising the emission frequencies of their discrete force elements. This synchronicity is proposed as the mechanism for the strong force and for coherent assemblies (Pons et al., 2013c). This is relevant because it will be shown that positronium can be interpreted within this theory as a type of synchronous-bound state.

\subsection{Positronium and Spin}

Positronium is the temporary bound states of electron-antielectron. Two states are known: parapositronium (life of about 125E-12 s), and orthopositronium (life 142E-9 s). Positronium has been relatively well studied (Hautojarvi \& Vehanen, 1979) and production channels modelled mathematically (Frolov et al., 1995; Lepage, Mackenzie, Streng, \& Zerwas, 1983). Positronium has the known behaviour of producing two photons when the electron and positron have antiparallel spins (parapositronium), and three photons for parallel spins (orthopositronium).

Spin in quantum theory refers to the quantised angular momentum of the particules. However this is physically undefined for a zero-dimensional point particule. Consequently quantum theory finds it difficult to explain how spin differences should affect lifetime for positronium. The Cordus theory, being a hidden-variable design, has an explanation for spin, which makes it possible to explain the different behaviours of positronium in a natural way. Since Cordus particules have span, they consequently have relative angular orientation, and phase differences. In this theory the spatial orientation of one particule relative to another is defined by the angular orientation of the fibrils, and the relative phases of energisation.

\section{Hand determines bonding vs. annihilation}

It is obvious that annihilation is not merely a charge effect, since an electron and a proton do not annihilate each other, but instead bond. The Cordus theory explains this as a consequence of the synchronous interaction. The free electron and proton exert constraints on each other via discrete forces, whereby they negotiate complementary emission directions and synchronised frequency thereof. Species from the same hand (e.g. matter and matter) do not annihilate, but can only share space and balance their charges at assembly, hence the bonding. Annihilation is proposed to occur when all the discrete forces of both particules are co-linear and complementary, in which case they may merge and then collapse their discrete forces into the photon types. This requires opposite charge and opposite hand. Thus a dexter electron and a sinister antielectron, when placed close together, can merge their discrete forces and transform back into photon energy from which they were made. It is the details of that process to which we now turn.

\subsection{Annihilation Process for Parapositronium}

It is known that in parapositronium the two particles have antiparallel spins. The life before annihilation is the shorter of the two forms. Annihilation is known to produce two photons, or less often 4 or 6 etc. The Cordus theory explains the para- or antiparallel spin state as a trans-phasic assembly of two particules. Trans-phasic means that two reactive ends, one from each of two particules, are adjacent and energising out of phase ( $\pi$ relative phase angle). The proposed collapse sequence is illustrated in Figure 4.

\section{Initial engagement}

When the e and e come within proximity, their discrete forces start to engage, well before the reactive ends themselves are close. This engagement aligns the two spans parallel and draws the reactive ends into geometric coincidence, see Figure 5. The mechanisms for this part of the process are electrostatic and magnetic forces mediated through the discrete forces. Subsequently the synchronicity takes hold, and the strong interaction arises. 


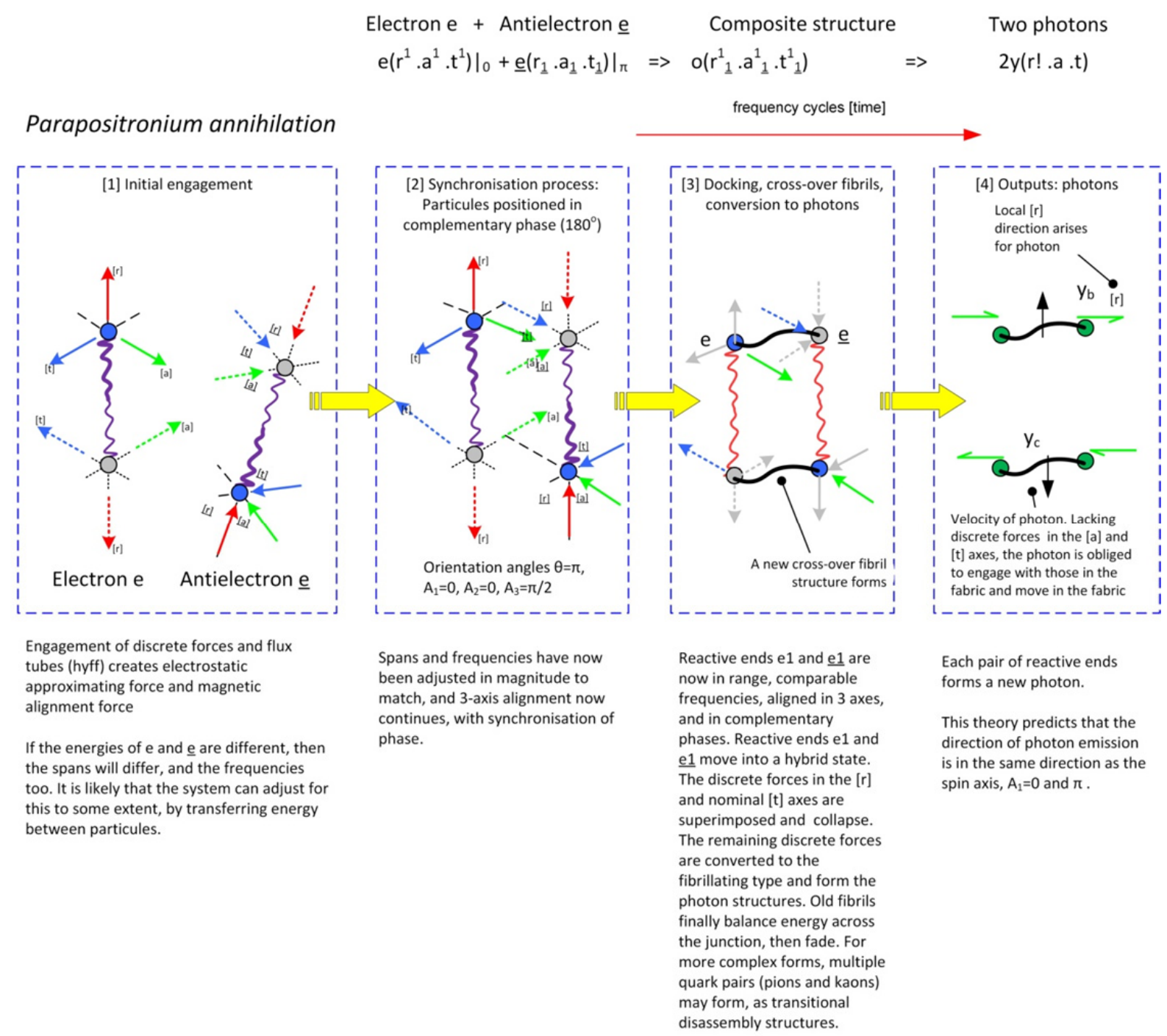

Figure 4. Annihilation for parapositronium electron and antielectron under the Cordus theory. Stage 1: Initial engagement of electron and antielectron in a process of mutual alignment. Stage 2: The electron and antielectron synchronise their frequencies. Photon emission may occur if necessary for synchronisation. Stage 3: Docking process involves the geometric alignment of the reactive ends and a growing interaction between the e1 and $\underline{e}$ reactive ends. Cross-over involves the formation of transverse fibrils. Stage 4: Reactive ends strengthen the transverse fibril links and the original fibrils decay, resulting in two output photons

\section{Synchronisation process.}

It is one thing for the participating particules to be near each other, and sufficiently aligned, but the next necessary step in the process, according to this theory, is synchronisation. This positions the particules in a complementary way to each other, i.e. trans-phasic. This also requires the frequency of the two particules to be sufficiently similar. The process of negotiating synchronicity automatically also achieves this. One particule may have higher energy (faster frequency), and the negotiations can cause it to slow its frequency and the other particule to increase frequency (Note 3). Thus the discrete forces accomplish energy transfer between particules. In this condition they are in a bound state, albeit temporary. We identify this as the bonding mechanism for positronium.

So the initial engagement is a process of geometric alignment, whereas the synchronisation is of frequency and its phase. We anticipate that the two processes occur concurrently, so our differentiation of them into distinct processes is for descriptive clarity rather than temporal accuracy. Another simplification is that the diagrams show one set of discrete forces as active (solid lines) and the other as inactive (dashed lines). However this should not be interpreted as implying a step on-off change between the two sides of the particule. Instead there is 
expected to be a progressive transition.

The particules operate at the discrete force level, and so each round of force and energy balancing requires another discrete force emission round, i.e. another frequency cycle. Frequency cycles are time in this theory, the two are indistinguishable (Pons et al., 2013a), and therefore the process of forging compatibility takes time. Consequently a testable prediction arises: This theory predicts that particules with greater disparity in energy or less degrees of freedom, will take longer to annihilate. Also, for cases where both particles have the same energy, higher-frequency is expected to result in faster reactions.

\subsection{Cordus Mechanics for Annihilation}

In the Cordus theory the type of particle is defined by the discrete forces it emits. To complete the explanation of annihilation we need to show how the discrete forces of the positronium assembly become rearranged to those of the photon. The following explanation is offered, see Figure 5.

\section{Details of the conversion to photons (stage 3)}

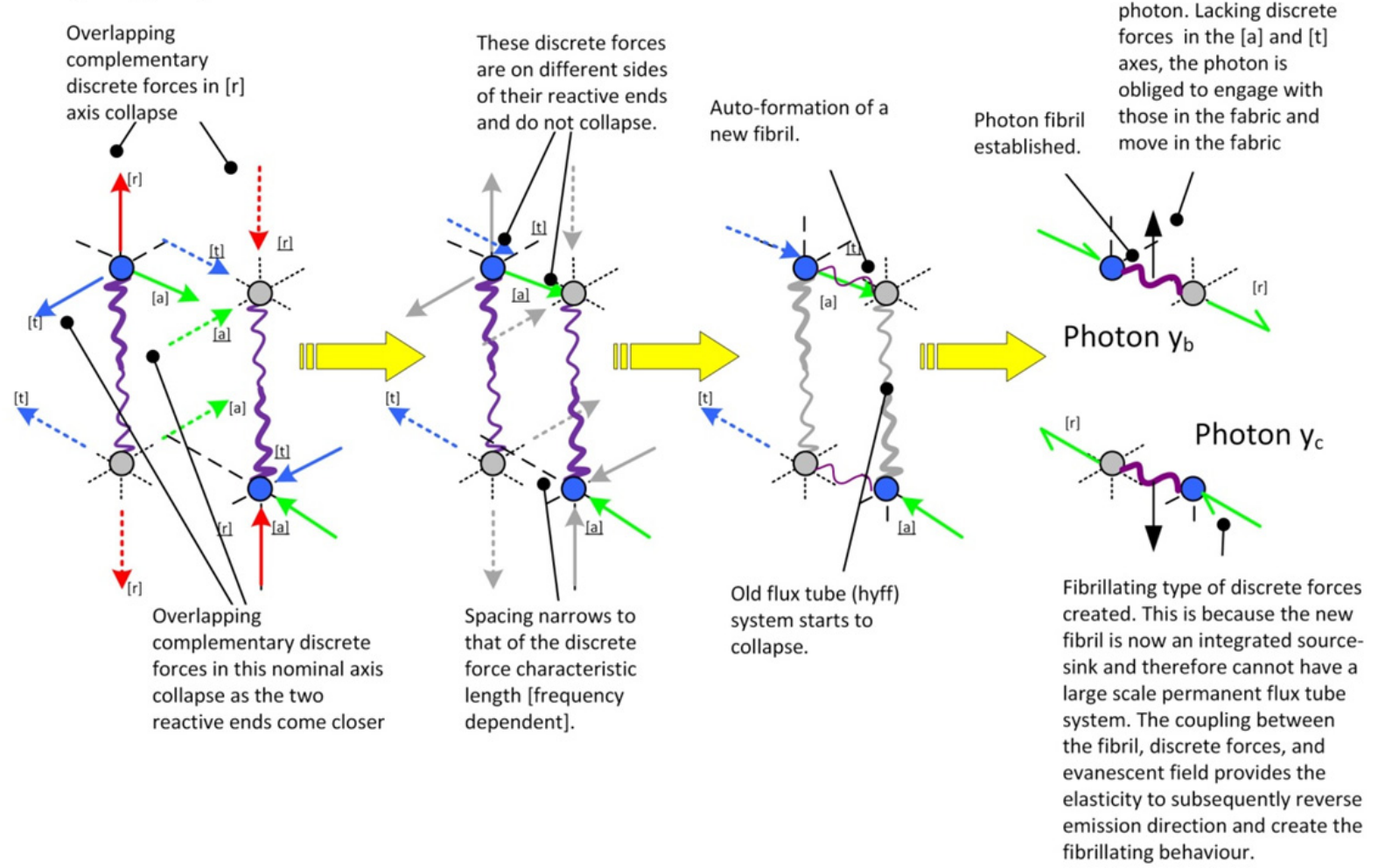

Figure 5. Conversion details for photons

Once the reactive ends are within range of each other, geometrically aligned, at complementary frequencies, and in phase then the process of annihilation gets underway. The increasingly overlap of the discrete forces causes a confused state of the flux tubes. This starts to take on some of the features of a fibril. Thus there is a growing connection between the $\mathrm{e} 1$ and $\underline{\mathrm{e}}$ reactive ends, i.e. an inter-action at the expense of the intra-action. The identities of the original participating particules become weaker, and a temporary square structure arises. This readies the system for the next transition. A new fibril forms between the e1 and el reactive ends when their discrete forces are sufficiently co-incident, co-linear, at the same frequency, and suitable phase. The original fibrils fade out. These had been of the pulsatile type: discrete force pulses moving in one direction. Also, the two reactive ends were out-of-phase $\left(180^{\circ}\right.$ phase offset), so that one reactive end was energised while the complementary one was not. In contrast the new fibril is the oscillating type: two discrete force pulses moving in the same direction, and then reversing. Both the new reactive ends are active at once (in-phase or $0^{\circ}$ offset). This is the structure of the photon. Thus the outcome of this process is a photon from each pair of reactive ends, 
shown as $\mathrm{y}_{\mathrm{b}}$ and $\mathrm{y}_{\mathrm{c}}$.

The conventional explanation for the production of two photons, rather than one, is that this is necessary for conservation of energy and momentum. The Cordus explanation is consistent with this, and extends to suggesting a mechanism: having four reactive ends involved requires that pairs of photons be produced. The reactive ends are more enduring structures than the discrete forces. The two photons $\mathrm{y}_{\mathrm{b}}$ and $\mathrm{y}_{\mathrm{c}}$ emerge simultaneously, not sequentially, in this version of the theory (Note 4). These two photons are predicted to be of opposite polarity (polarisation) but identical energy. The polarity arises because the original participating particules were of the oscillating frequency type $\left(180^{\circ}\right.$ phase). The identical energy arises because (a) the initial synchronisation process balances the energy between the electron and antielectron, and (b) the fibrils distribute and balance the energy between the reactive ends. So there is a balancing of energy across all four reactive ends involved, and this carries forward to the photons. Consequently a testable prediction arises:

This theory predicts that the two photons emerging from parapositronium annihilation will have the following characteristics: (a) be emitted in opposite directions along the parapositronium spin axis, (b) have opposite $(\pi)$ polarisation, (c) be identical in energy.

The very last stage, whereby the photon's discrete forces reverse direction at the next energy cycle, is proposed to be a consequence of the dynamic coupling between discrete forces and evanescent field set up in the medium. The photon cannot release its discrete force into the fabric, unlike the electron, because it has an integrated source-sink arrangement for its two reactive ends. Consequently the propagation of the discrete force is pushed one way into the fabric, elastically recoils from the fabric, and reverses direction. The fibril allows the two discrete forces to be instantly coordinated, so that what happens at one reactive end also happens at the other, or at least the complementary action occurs, because the discrete forces are in different directions relative to the reactive end. (Time does not exist within the fibril, because time is only generated at the next level up, which is the frequency oscillations of the particule as a whole.) If there is sufficient energy then additional photons or other transitional particules may be produced at this stage by the production of complementary discrete forces pairs and their allocation to particules.

\section{Parapositronium annihilation in HED notation}

Note that in the above process diagram the horizontal axis is time. More specifically, the Cordus theory identifies that time at the deeper level corresponds to the re-energisation frequency cycles of the particules (Pons et al., 2013a). Thus particules need cycles to accomplish the process activities, hence annihilation is not instantaneous. The parapositronium process is represented in HED notation:

$\left.\mathrm{e}\left(\mathrm{r}^{1} \cdot \mathrm{a}^{1} \cdot \mathrm{t}^{1}\right)\right|_{0 \mathrm{deg}}+\left.\underline{\mathrm{e}}\left(\mathrm{r}_{\underline{1}} \cdot \mathrm{a}_{\underline{1}} \cdot \mathrm{t}_{\underline{1}}\right)\right|_{180 \mathrm{deg}}$

$\Rightarrow \mathrm{o}\left(\mathrm{r}^{1} \cdot \mathrm{a}_{1}^{1} \cdot \mathrm{t}_{1}^{1}\right)$

$\left.\Rightarrow y_{b}(r$ ! .a.$t)\right|_{0 \text { deg }}+\left.y_{c}(r$ ! .a.t $)\right|_{180 \text { deg }}$

$\Rightarrow \mathrm{y}_{\mathrm{b}}+\mathrm{y}_{\mathrm{c}}$

where "o" represents a transitional state. In this particular case, this can be identified as parapositronium. Thus an electron and antielectron in parapositronium annihilate to two photons.

\subsection{Orthopositronium}

Orthopositronium is the other temporary association of an electron and antielectron, and has the longer life before annihilation, though still short. It is known that the two particles have parallel spins. Annihilation is known to produce three photons, less often five. One of the photons may be of a different energy (Manohar \& Ruiz-Femenia, 2004).

The Cordus theory qualitatively explains the longer life and production of an odd number of photons. First it is necessary to refer back to the Cordus theory for spin, and note that parallel spin corresponds to two particules the fibrils of which are oriented the same in space, and with energisation phase $\theta=0$ (cisphasic). This state permits alignment between the discrete forces of neighbouring reactive ends. We anticipate this situation arises either because it is geometrically closest for the two particules, or the particules do not have sufficient degrees of freedom to rotate. In turn that might be due to other bonding commitments, or too much momentum to be able to make the necessary adjustment manoeuvre in the time available.

The orthopositronium state provides a certain degree of bonding, but this is unstable because the energisation sequences (hands) are different. The relative orientation of the two particules needs to change: one of them needs to rotate. (We nominally show the antielectron doing all the adjustment, but this is merely for convenience or representation). 


\section{Photon-emission phase-offset}

For this design to work requires a further and very specific assumption, that emission of a photon causes a particule to delay the re-energisation of its reactive end by half a frequency cycle, i.e. to change its phase $\theta$ by $\pi$. Thus a particule-pair that is caught in a certain unfavourable phase state may escape that state by emitting a photon. We anticipate that either the electron or antielectron may emit the photon, and that it will probably be whichever is more geometrically constrained or higher energised. With that addition it possible to explain the orthopositronium behaviour, see Figure 6 .

We record this assumption as a lemma (see Appendix), thereby indicating that it is a logical necessity of this design. Noting these lemmas is important for the overall coherence of the theory, as they provide a logical interlocking of the sub parts of the theory. The lemmas also indicate the vulnerable areas of the theory, since disproving a lemma will falsify part of the theory.

This idea that a change in energisation phase is possible by emitting a photo might seem to be an ad-hoc contrived postulate in an already conjectural theory. Nonetheless the proposed effect is consistent with the Sokolov-Ternov effect whereby electrons or antielectrons can invert their spin by synchrotron radiation.

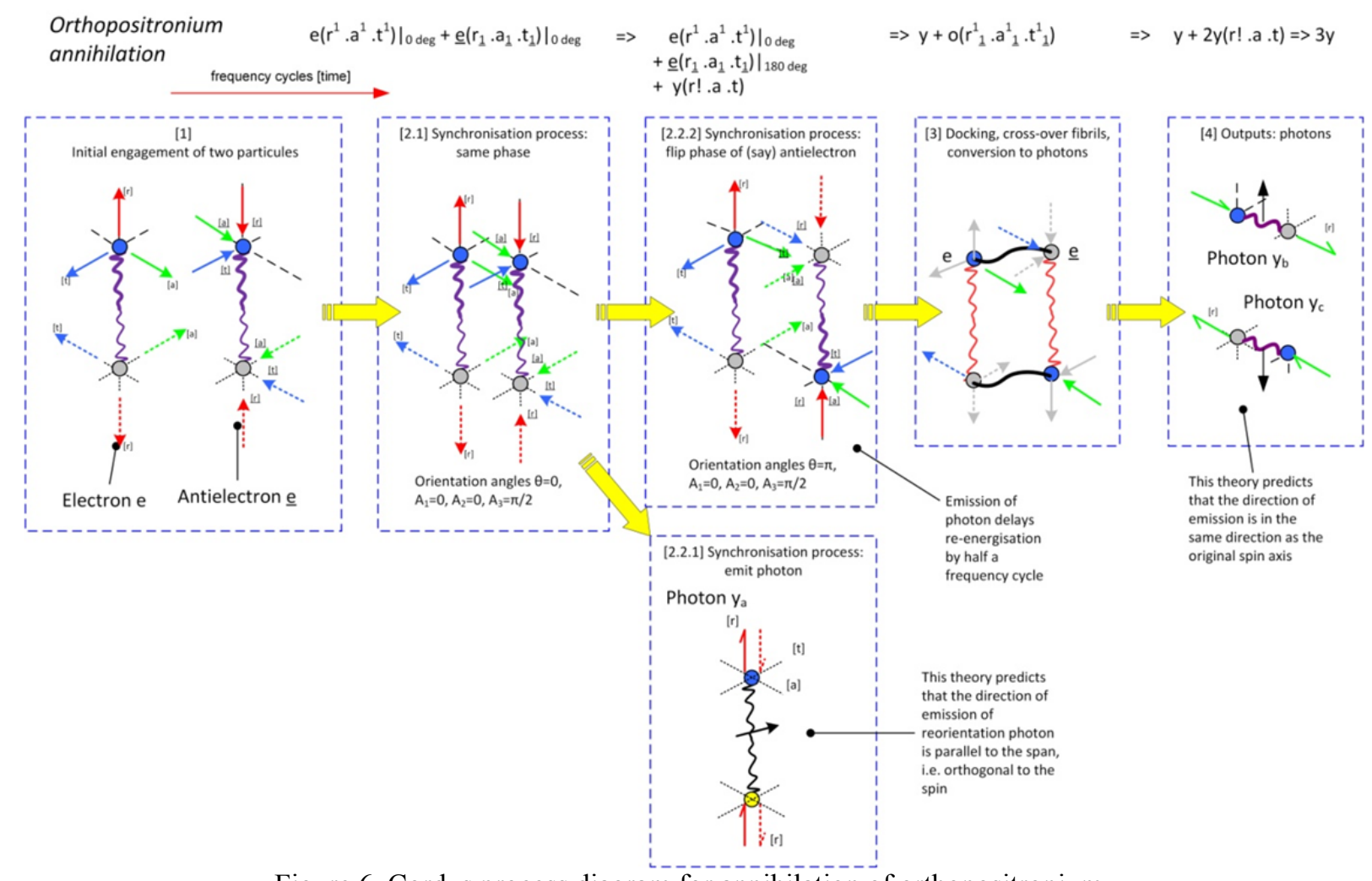

Figure 6. Cordus process diagram for annihilation of orthopositronium

This diagram is more complex than the previous one. This is because orthopositronium has additional activities required before the main annihilation process can get underway. A testable prediction arises: That the first (of three) photons emerging from orthopositronium annihilation will have the following characteristics: (a) be emitted before the other two, (b) be emitted orthogonal to the spin axis of the orthopositronium assembly, (c) be dissimilar in energy to the other two photons.

The short-hand representation of this in the HED notation is:

$$
\begin{aligned}
& \left.\mathrm{e}\left(\mathrm{r}^{1} \cdot \mathrm{a}^{1} \cdot \mathrm{t}^{1}\right)\right|_{0 \text { deg }}+\left.\underline{\mathrm{e}}\left(\mathrm{r}_{\underline{1}} \cdot \mathrm{a}_{1} \cdot \mathrm{t}_{1}\right)\right|_{0 \mathrm{deg}} \\
& =>\mathrm{y}_{\mathrm{a}}(\mathrm{r} ! \cdot \mathrm{a} \cdot \mathrm{t})+\left.\mathrm{e}\left(\mathrm{r}^{1} \cdot \mathrm{a}^{1} \cdot \mathrm{t}^{1}\right)\right|_{0 \mathrm{deg}}+\left.\underline{\mathrm{e}}\left(\mathrm{r}_{\underline{1}} \cdot \mathrm{a}_{\underline{1}} \cdot \mathrm{t}_{\underline{1}}\right)\right|_{180 \mathrm{deg}} \\
& =>\mathrm{y}_{\mathrm{a}}+\mathrm{o}\left(\mathrm{r}^{1}{ }_{\underline{1}} \cdot \mathrm{a}^{1} \cdot \mathrm{t}_{\underline{1}} \cdot \mathrm{t}^{1}\right) \\
& \Rightarrow>\mathrm{y}_{\mathrm{a}}+\left.\mathrm{y}_{\mathrm{b}}(\mathrm{r} ! \cdot \mathrm{a} \cdot \mathrm{t})\right|_{0 \mathrm{deg}}+\left.\mathrm{y}_{\mathrm{c}}(\mathrm{r} ! \cdot \mathrm{a} \cdot \mathrm{t})\right|_{180 \mathrm{deg}}
\end{aligned}
$$


$\Rightarrow \mathrm{y}_{\mathrm{a}}+\mathrm{y}_{\mathrm{b}}+\mathrm{y}_{\mathrm{c}}$

Or in the reduced format:

$\mathrm{e}+\underline{\mathrm{e}}=>3 \mathrm{y}$

Thus an electron and antielectron in orthopositronium annihilate to three photons. The reason orthopositronium cannot emit only two photons is conventionally explained as a consequence of charge conjugation invariance. (Charge conjugation invariance is the expectation that process, such as the emission of photons, are the same -hence invariant- if all the particles are replaced with antiparticles). From the Cordus perspective the reason is complementary but expressed differently: that emission of one photon is required to change the spin state to antiparallel, and the conservation of reactive ends requires that at least two photons be produced subsequently.

\subsection{Comparison: Parapositronium vs. Orthopositronium}

The Cordus theory predicts that the two- and three-photon production processes for para- and ortho-positronium are very different: the third photon is not merely one of three, but has a different causality and comes out at a different part of the process. It is proposed that both forms of positronium use the same core annihilation process (stages 3-4) for the production of the paired photons.

The Cordus theory also offers a qualitative explanation of why the lifetime for parapositronium is so much less than orthopositronium: the latter has further processes to undergo, and these take time. Parapositronium is a preassembly that is already in a suitable orientation to proceed to photon production. By comparison orthopositronium first has to emit a photon before it can continue. If this interpretation is correct, then we can make another inference: that the time taken to get the particules into the correct geometric position is much the greater contributor to the decay time than the annihilation process to photons.

These explanations for the production of two and three photons are consistent with known behaviour of positronium. The Cordus theory also independently derives the spin requirement.

\section{Various output photon scenarios}

The annihilation of an electron and antielectron is known to produce two photons (or less often 4, 6..) or three (less often 5). It is known to depend on the relative spins: antiparallel or parallel spins respectively. Output of a single photon is possible, but only if there is other matter nearby to absorb some of the energy. The Cordus theory explains these outcomes as follows:

- One photon. Single photon, nominally $y_{b}$, is emitted. Its companion $y_{c}$ is emitted and immediately absorbed by nearby matter (e.g. other electrons) before detection. This effect may also remove photons from any of the following cases.

- Two photons, $\mathrm{y}_{\mathrm{b}}$ and $\mathrm{y}_{\mathrm{c}}$ are produced from each pair of reactive ends. This occurs if the original e and $\underline{\mathrm{e}}$ were in opposite energisation $\left(180^{\circ}\right.$ phase offset).

- Three photons. The first photon, $\mathrm{y}_{\mathrm{a}}$ is produced as an initial adjustment to get the e and e into in a suitable phase. The $\mathrm{y}_{\mathrm{b}}$ and $\mathrm{y}_{\mathrm{c}}$ photons are subsequent outcomes.

- Four, six, or higher even counts of photons. This is an extension of the two-photon model, where transitional structures (e.g. more electron-antielectron pairs) form at stage \#3 due to the energy content of the original electron and antielectron.

- Five or higher odd counts of photons. This is an extension of the three-photon model, with additional pair production at stage \#3 cross-over, again due to available energy.

\subsection{Bhabha Scattering}

While the annihilation an electron and antielectron has been the primary focus of this paper, their interaction can instead result in elastic recoil, hence Bhabha scattering. This scattering has been thoroughly modelled mathematically, but still the ontological question remains: Why does annihilation not always occur? Since the mechanisms are strong enough to annihilate the pair, what defeats them so that scattering can take place? We believe a simple qualitative explanation is available. It is that particules interact through their discrete forces as they approach each other. The flux tubes have to negotiate mutual emission directions (HEDs) and thus they exert force on each other before the reactive ends actually coincide. The reaction forces occur at a small distance away from the reactive end. (This is a non-local theory, in which the conventional principle of locality at a point is replaced by a principle of Wider Locality.) Thus the scattering outcome ultimately depends on the frequency, phase, and the orientation of the particules. In the specific case of Bhabha scattering, it is proposed that there are two contributory factors: if the phase is mismatched then the synchronous interaction causes repulsion, and if the 
momentum is too high the particules do not have enough frequency cycles (time) to get into a complementary phase state. This is represented in Figure 7.

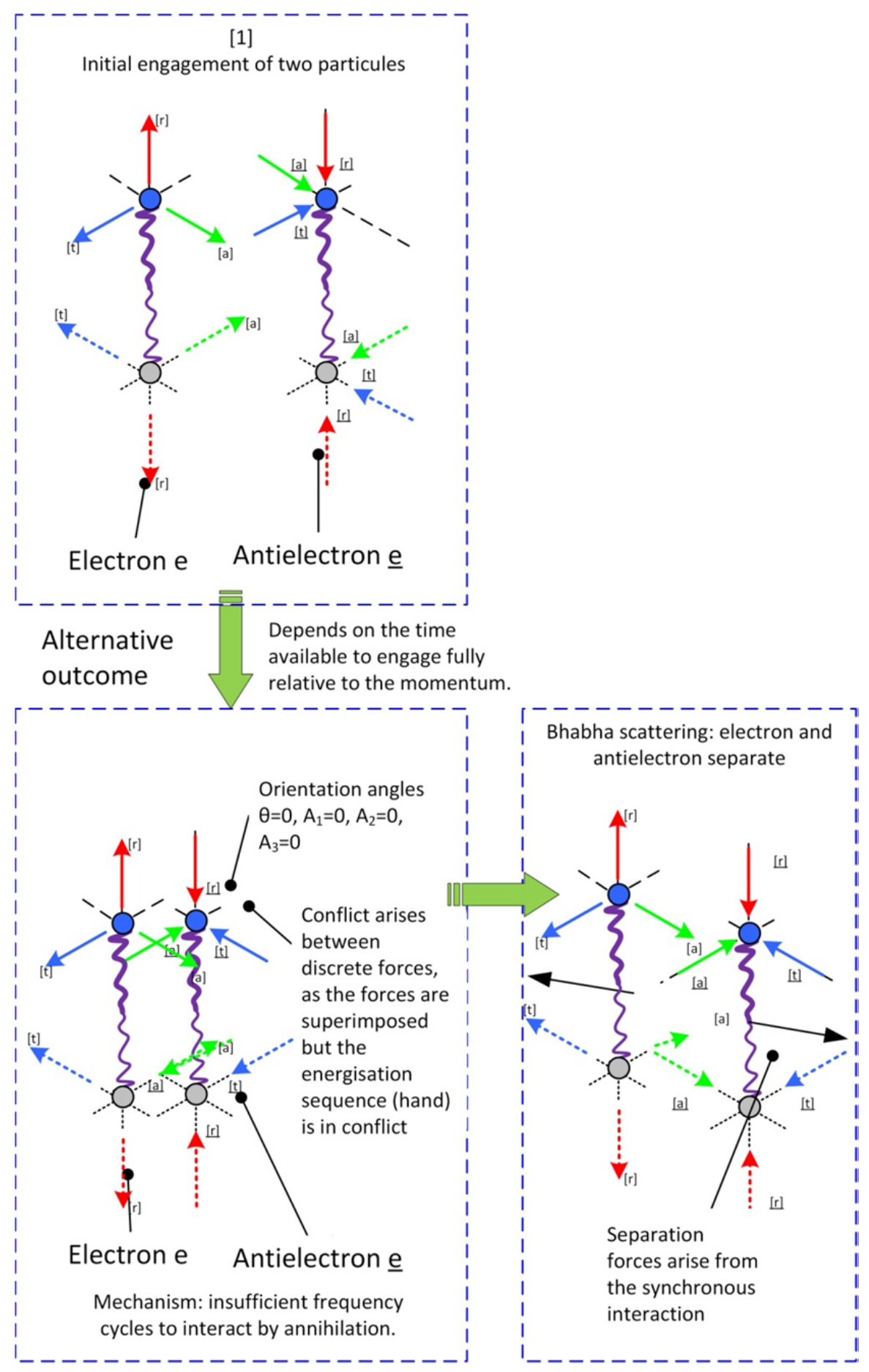

Figure 7. Explanation for Bhabha scattering

\section{Discussion}

\subsection{What Has Been Achieved?}

This work has made several novel intellectual contributions. The first is the presentation of an ontologically rich explanation of the inner mechanics of the annihilation process. This explains the process in terms of the handedness of matter and antimatter, the interaction of the two particules as they approach, the collapse of their 
discrete force structures and their reformation into photon structures.

A second contribution is provision of a theory that explains why para- and ortho-positronium produce different quantities of photons at annihilation. Furthermore, the theory qualitatively explains the relative difference in lifetimes. There is also provision of a qualitative explanation for why Bhabha scattering rather than annihilation sometimes occurs.

A third contribution is methodological, in that it has been demonstrated that the hidden-variable sector, which is otherwise under-appreciated, has the potential to provide plausible solutions to complex foundational problems. We have shown how a systematic design method can construct solutions in this sector. This is novel given that design has otherwise not been explicitly used this way in physics. A related contribution is the provision of a logically consistent theory with high explanatory power across a wide-range of physical phenomena.

The theory also has implications at the cosmological level, e.g. horizon effect (D. J. Pons \& A. D. Pons, 2013), a comprehensive theory of time (Pons et al., 2013a), and theory for the stability of the nuclides ( $\mathrm{H}$ to $\mathrm{Ne}$ ) (Pons et al., 2013b).

\subsection{What Are the Implications?}

\section{Other annihilation productions}

The area under examination has been the annihilation process between an electron and antielectron (positron). According to this theory, the electron is an ideal particule since it has low rest mass (hence easy to manufacture in pair production), simple discrete force structures (hence compatible with many other particules including other electrons -whereas protons are incompatible with other protons), and is flexible about the energies, hence frequency, it adopts. The same reasons also cause the electron-antielectron annihilation process to be a relatively clear progression to photons, whereas nucleon annihilation involves a more complex set of intermediate mesons. There is reason to believe that the mechanics proposed for electron-antielectron annihilation are scalable to other annihilation situations. The implications are that it could be useful when considering meson behaviour, e.g. pions and kaons, to apply the concept of discrete forces.

\section{Complementarity of bonding and annihilation}

The Cordus theory suggests that strong-force bonding and annihilation share the same deeper mechanics. Thus it is proposed that same-hand particules can bond, whereas contrary handed particules can annihilate (Pons et al., 2013c). Bonding and annihilation become complementary processes for ipsilateral and contralateral handedness respectively. The common deeper mechanics is an interaction between discrete forces from neighbouring particules. This interaction also explains scattering. Thus it is possible to conceive of a deeper conceptual framework wherein these three very different phenomena are unified.

\section{What happens to the information at annihilation?}

In quantum mechanics the information contained in matter, such as its quantum numbers, cannot vanish. The Cordus theory offers a different interpretation, as follows. Before the particules annihilate they are sending out electro-magneto-gravitational (EMG) discrete forces into the surrounding space, advertising their existence and affecting other remote particules. The discrete forces propagate distally at the speed of light. Thus a remote mass may become aware of one of the particules, and an EMG force, say of gravitational attraction, starts to act. (Force in this theory is interpreted as a discrete prescribed constraint on re-energisation position of the reactive end, i.e. an incremental displacement effect.) At the moment of annihilation the production of new discrete forces ceases. What then happens to the particule's responsibility to the remote mass? The answer, according to this view of events, is that the existing discrete forces that are in-transit continue to propagate outwards, and the remote mass continues to respond to the force while those discrete forces continue to be supplied. When the flow ceases then the force also ceases. So the remote mass continues to feel the force after the particules have annihilated. Thus the information about the cessation in production also travels outward at the speed of light. Knowledge of the existence of the two annihilated particules is thus progressively wiped from the universe.

So the Cordus theory suggests that the information about the electron and antielectron does vanish, being replaced by photons with some of the information (e.g. coded in the emission direction and phase), but not necessarily all. Another way to consider the matter is that the initial process of genesis, which manufactured photons into electrons and antielectrons, introduced variables that were merely temporary anyway. The production of multiple photons (4 or more) from energetic electron-antielectron annihilation events suggests that conservation of quantum numbers may not be the best way to look at the problem anyway. The Cordus theory suggests instead that particules are defined by their discrete force structure, and if there is sufficient energy to create those structures, then the particule will come into existence. The focus is therefore on discrete forces as 
opposed to quantum numbers of point particles.

\subsection{Limitations}

We acknowledge that the theory has not described the deeper physics of how the proposed reactive ends, discrete forces, flux tubes, and fibrils operate. All that we can say is to reiterate the design perspective: that if particules were to have these structures then it logically follows that annihilation and many other effects can be explained.

A common criticism of the Cordus theory is the lack of a quantitative framework or mathematical formalism to the work. This would be a valid criticism if the objective was to incrementally improve an existing mathematical framework like quantum theory or string theory. However that is not our objective: we do not seek to refine but to reconceptualise. From a design perspective this theory is still in the divergent conceptual phase, and the detailed convergence is a future endeavour. Thus the focus at this stage in the life-cycle of the theory is to create a broad conceptual foundation and check that it is logically consistent across a wide range of phenomena. The quantitative solution and mathematical formalism is a detailed activity that we leave for future work.

\subsection{Implications for Further Research}

This theory suggests several lines of future enquiry. The pair-production mechanisms appear to be simply the reverse of the annihilation process described here, but this needs to be checked. A future endeavour could be the development of a mathematical formalism. Another is to explore the formation processes for the mesons and the production of neutrinos.

The theory identifies specific testable hypotheses:

- Particules with greater disparity in energy or less degrees of freedom, will take longer to annihilate. Also, for cases where both particles have the same energy, higher-frequency is expected to result in faster reactions.

- The two photons emerging from parapositronium annihilation will have the following characteristics: (a) be emitted in opposite directions along the parapositronium spin axis, (b) have opposite $(\pi)$ polarisation, (c) be identical in energy.

- The first (of three) photons emerging from orthopositronium annihilation will have the following characteristics: (a) be emitted before the other two, (b) be emitted orthogonal to the spin axis of the orthopositronium assembly, (c) be dissimilar in energy to the other two photons.

\section{Conclusions}

The original purpose was to explain annihilation from first principles. This has been achieved, starting from the discrete forces of a non-local hidden-variable (NLHV) design. This explains the process in terms of the handedness of matter and antimatter, the interaction of the two particules as they approach, the collapse of their discrete force structures and their reformation into photon structures. The resulting theory successfully explains para- and ortho-positronium annihilation. It explains the different photons output, the relative difference in lifetimes, and why Bhabha scattering sometimes happens instead.

This theory also addresses the deeper ontological question of why annihilation happens at all. The answer is that matter and antimatter are segregated forms of energy -segregated by hand- and annihilation is simply the reversal of that process. At a deeper level there is a conservation at work, one of discrete forces and reactive ends and energy, so that the process is better thought of as remanufacture as opposed to a destructive loss.

\section{References}

Abak, M. (1976). S-matrix theory of weak interactions and the electron-positron pair annihilation process e-+e+N+N. Lettere al Nuovo Cimento, 16(8), 245-251. http://dx.doi.org/10.1007/BF02747029

Adachi, K., Hayashii, H., Miyabayashi, K., Noguchi, S., Miyamoto, A., Tauchi, T., ... Yamauchi, M. (1999). Measurement of the jet width in collisions and in e+e- annihilation process at TRISTAN. Physics Letters B, 451(1-2), 256-266. http://dx.doi.org/10.1016/S0370-2693(99)00142-2

Bardin, D., \& Riemann, T. (1996). Off-shell W pair production in e+e- annihilation: the CC11 process. Nuclear Physics B, B462(1), 3-28. http://dx.doi.org/10.1016/0550-3213(95)00645-1

Brandelik, R., Braunschweig, W., Gather, K., Kadansky, V., Lubelsmeyer, K., Mattig, P., ... Zobernig, G. (1980). Comparison of e+e- annihilation with QCD and determination of the strong coupling constant. Physics Letters B, 94(3), 437-443. http://dx.doi.org/10.1016/0370-2693(80)90914-4 
Chao, W., Meng, T., \& Pan, J. (1986). What does the observed rapidity dependence of the multiplicity distribution in e+e- annihilation processes tell us? Physics Letters B, 176(1-2), 211-214. http://dx.doi.org/10.1016/0370-2693(86)90952-4

Field, R. D., \& Wolfram, S. (1983). A QCD model for e+e- annihilation. Nuclear Physics B, 213(1), 65-84. http://dx.doi.org/10.1016/0550-3213(83)90175-X

FIPS. (1993). Integration Definition for Function Modeling (IDEF0). Retrieved 12 Aug, 2003, from http://www.itl.nist.gov/fipspubs/idef02.doc

Frolov, A. M., Kryuchkov, S. I., \& Smith, V. H., Jr. (1995). (e-,e+)-pair annihilation in the positronium molecule Ps2. Physical Review A (Atomic, Molecular, and Optical Physics), 51(6), 4514-4519. http://dx.doi.org/10.1103/PhysRevA.51.4514

Gronau, M., Smith, C. H. L., Walsh, T. F., Wolfram, S., \& Yang, T. C. (1977). Lepton energy spectra in e+eannihilation and other processes. Nuclear Physics B, B123(1), 47-60. http://dx.doi.org/10.1016/0550-3213(77)90339-X

Hautojarvi, P., \& Vehanen, A. (1979). Introduction to positron annihilation Positrons in solids (pp. 1-23). Berlin, West Germany: Springer-Verlag.

Honda, Y., Shimada, T., Tashiro, M., Kimura, N., Yoshida, Y., Isoyama, G., \& Tagawa, S. (2007). Study of annihilation processes of positrons in polystyrene-related polymers. Radiation Physics and Chemistry, 76(2), 169-171. http://dx.doi.org/10.1016/j.radphyschem.2006.03.029

Jean, P., Knodlseder, J., Gillard, W., Guessoum, N., Ferriere, K., Marcowith, A., ... Roques, J. P. (2006). Spectral analysis of the Galactic e+e- annihilation emission. Astronomy \&amp; Astrophysics, 445(2), 579-589. http://dx.doi.org/10.1051/0004-6361:20053765

Jonsell, S., Saenz, A., Froelich, P., Zygelman, B., \& Dalgarno, A. (2001). Stability of hydrogen-antihydrogen mixtures at low energies. Physical Review A (Atomic, Molecular, and Optical Physics), 64(5), 052712-052711. http://dx.doi.org/10.1103/PhysRevA.64.052712

Kazama, Y., \& York-Peng, Y. (1979). High-energy inclusive annihilation processes. II. Quantum chromodynamics. Physical Review D (Particles and Fields), 19(10), 3121-3134. http://dx.doi.org/10.1103/PhysRevD.19.3121

Kurihara, Y., Fujimoto, J., Munehisa, T., \& Shimizu, Y. (1996). Hard photon distributions in e+e- annihilation process by QEDPS. Progress of Theoretical Physics, 96(6), 1223-1235. http://dx.doi.org/10.1143/PTP.96.1223

Lampe, B., \& Kramer, G. (1983). Application of Gegenbauer integration method to e+e- annihilation process. Physica Scripta, 28(6), 585-592. http://dx.doi.org/10.1088/0031-8949/28/6/002

Lepage, G. P., Mackenzie, P. B., Streng, K. H., \& Zerwas, P. M. (1983). Multiphoton decays of positronium. Physical Review A, 28(5), 3090. http://dx.doi.org/10.1103/PhysRevA.28.3090

Lyuboshitz, V. (2008). Spin correlations of muons in the process of electron-positron pair annihilation e+e- + European Physical Journal Special Topics, 162, 43-51. http://dx.doi.org/10.1140/epjst/e2008-00774-y

Lyuboshitz, V. (2009). On the spin correlations of muons generated in the annihilation process e+e-+. Physics of Atomic Nuclei, 72(2), 311-318. http://dx.doi.org/10.1134/S1063778809020173

Manohar, A. V., \& Ruiz-Femenia, P. (2004). Orthopositronium decay spectrum using NRQED. Physical Review D, 69(5), 053003:053001-053011. http://dx.doi.org/10.1103/PhysRevD.69.053003

Pei, Y. -J. (1996). A simple approach to describe hadron production rates in $\mathrm{e}^{+} \mathrm{e}^{-}$annihilation. Zeitschrift für Physik C Particles and Fields, 72(1), 39-46. http://dx.doi.org/10.1007/s002880050221

Pons, D. J., \& Pons, A. D. (2013). Outer boundary of the expanding cosmos: Discrete fields and implications for the holographic principle The Open Astronomy Journal, 6, 77-89. http://dx.doi.org/10.2174/1874381101306010077

Pons, D. J., Pons, A., D., \& Pons, A., J. (2013a). Time: An emergent property of matter. Applied Physics Research, 5(6), 23-47. http://dx.doi.org/10.5539/apr.v5n6p23

Pons, D. J., Pons, A. D., \& Pons, A. J. (2013b). Explanation of the Table of Nuclides: Qualitative nuclear mechanics from a NLHV design. Applied Physics Research, 5(6), 145-174. http://dx.doi.org/10.5539/apr.v5n6p145 
Pons, D. J., Pons, A. D., \& Pons, A. J. (2013c). Synchronous interlocking of discrete forces: Strong force reconceptualised in a NLHV solution Applied Physics Research, 5(5), 107-126. http://dx.doi.org/10.5539/apr.v5n5107

Pons, D. J., Pons, A. D., \& Pons, A. J. (2013, accepted). Differentiation of Matter and Antimatter by Hand: Internal and External Structures of the Electron and Antielectron. Physics Essays, Submission 29 May $2013>$ Receipt 8 June 2013 PE301209/01/EP/ep, 1-18.

Pons, D. J., Pons, A. D., Pons, A. M., \& Pons, A. J. (2012). Wave-particle duality: A conceptual solution from the cordus conjecture. Physics Essays, 25(1), 132-140. http://dx.doi.org/10.4006/0836-1398-25.1.132

Renard, F. M. (1976). Threshold effects in annihilation processes. Lettere al Nuovo Cimento, 16(17), 519-524. http://dx.doi.org/10.1007/BF02720395

\section{A. Appendix: Annihilation Lemmas}

The following lemmas summarise the assumptions in this annihilation theory, and the principles of the proposed mechanics.

1 Cordus principle of Convergent hyff emission: Annihilation occurs when the discrete forces of two separate particules are, at their adjacent reactive ends, pumping in the same absolute direction but from opposite sides of the reactive end.

2 In this model we define a suitable complementary phase for the annihilation of electron and antielectron as opposite, i.e. when the reactive end of the one particule is active while that of the other particule is dormant, i.e. 180 degree phase offset. It may take frequency cycles to accomplish this, hence time.

3 Cordus Principle of photon-emission phase-offset: emission of a photon from a particule will delay the re-energisation of its reactive end by half a frequency cycle, i.e. change its phase by $180^{\circ}$. This is equivalent to flipping the QM spin.

4 A fibril is formed between reactive ends when their hyff flux tubes are sufficiently co-incident, co-linear, at the same frequency, and suitable phase.

5 When hyff flux tubes form such a fibril, they can change from the pulsatile type (e.g. electron) to the oscillating type of the photon.

6 Cordus principle of Complementarity of bonding and annihilation. Bonding and annihilation are complementary processes for same- and contrary-handedness respectively.

6.1 Same-hand structures can interact to form bonds, by sharing hyff emission directions.

6.1.1 When the charges are the same (++ or - -) then the particules can co-exist, but only providing they also take opposite phase in their frequency cycles. Hence the Pauli Exclusion Principle for electrons. If they are in phase then electrostatic repulsion results.

6.1.2 For opposite charges (+ -) the particules form attractive interactions (bonds) when the reactive ends are in phase with each other (electrostatic attraction).

6.1.3 Annihilation is not available for same-hand particules.

6.2 Discrete forces from contrary handed particules can interact.

6.2.1 Particles can annihilate by merging hyff emission directions. However they have to align and get into complementary $180^{\circ}$ phase, and this make take frequency cycles and hence time.

6.2.2 Particles can form bonded structures, at least temporarily, when they are in phase with each other. Hence positronium, kaons, and other exotic mesons.

7 Cordus principle that hyff function defines particule form. The hyff functional variables are identified as: the quantity of hyff (charge), their direction (sign of charge), direction in the [r,a,t] axes (hyff emission directions, HEDs), phase offset across the two reactive ends (pulsatile vs. oscillating), and hand (energisation sequence). These factors determine what the particule will be, thus its form.

8 Hyff flux tubes are conserved in annihilation and bonding, though complementary hyff may collapse each other. If a new hyff is created then a complementary hyff is also created.

9 The annihilation process itself is fast (125E-12 s for parapositronium), whereas the geometric pre-positioning is relatively slower. 


\section{Notes}

Note 1. A process requires a production mechanism: While the term 'process' is often applied to mathematical models of annihilation, this is inaccurate, at least from a production engineering perspective, because the mechanisms that give rise to the outputs are still unknown. It is like watching the assembly of a motor car from a distance, so that the overall phases can be discerned, but not the tools, parts, and operating procedures.

Note 2. Inner and outer structure of the Cordus particule: The basic idea is that every particule has two reactive ends, which are a small finite distance apart (span), and each behave like a particle in their interaction with the external environment. A fibril joins the reactive ends and is a persistent and dynamic structure but does not interact with matter. It provides instantaneous connectivity and synchronicity between the two reactive ends. Hence it is a non-local solution: the particule is affected by more than the fields at its nominal centre point, and a principle of Wider Locality applies. Each reactive end of the particule is energised in turn at the frequency of that particule (which is dependent on its energy). The reactive ends are energised together for the photon, and in turn for matter particules. The frequency corresponds to the de Broglie frequency. The span of the particule shortens as the frequency increases, i.e. greater internal energy is associated with faster re-energisation sequence (hence also faster emission of discrete force and thus greater mass). When the reactive end is energised it emits discrete forces in up to three orthogonal directions. The quantity and direction of these are characteristic of the type of particule (photon, electron, proton, etc.), and the differences in these signatures is what differentiates the particules from each other. Although for convenience we use the term discrete force for these pulses, the Cordus theory requires them to have specific attributes that are better described as latent discrete prescribed displacements. This is because a second particule that subsequently receives one is prescribed to energise its reactive end in a location that is slightly displaced from where it would otherwise position itself. Thus in the Cordus theory, that which we perceive as force is fundamentally the effect of many discrete prescribed displacements acting on the particules, a kind of coercive displacement. These discrete forces are connected in a flux line that is emitted into the external environment. (In the Cordus theory this is called a hyperfine-fibril, or hyff). Each reactive end of the particule emits three such orthogonal hyff, at least in the near-field. The exception is the photon, which only emits radially. These directions are relative to the orientation of the span, and the velocity of the particule, and termed hyperfine-fibril emission directions (HEDs). The axes are named [r] radial outwards co-linear with the span, [a] and [t] perpendicular to the span and to each other. These are so-named for consistency with our previous nomenclature for the photon, but when applied to massy particules do not necessarily imply motion. It is proposed that the quarks and other leptons follow the same pattern, though in the case of the quarks not all the hyff emission directions $[\mathrm{r}, \mathrm{a}, \mathrm{t}]$ are filled (hence their fractional charge). In this theory electric charge is carried at $1 / 3$ charge per discrete force, with the sign of the charge being determined by the direction of the discrete force element. So the number and nature of energised HEDs determines the overall electric charge of the particule. The aggregation of discrete forces from multiple particules creates the EMG fields, which are thus discrete. The combined emission discrete forces makes up a 3-D composite structure. The direct lineal effect of the discrete force provides the electrostatic interaction, the bending of the hyff flux line provides magnetism, the torsion provides gravitation interaction, and the synchronicity between discrete force elements of neighbouring particules provides the strong force. These are all carried simultaneously by the composite discrete force element as it propagates outwards on the hyff flux. Assembled massy particules compete spatially for emission directions, and may synchronise their emissions to access those spaces. Thus there is mutual negotiation in the near-field between interacting particules, based on shared geometric timing constraints. These particules interact by negotiating complementary HEDs and synchronising the emission frequencies of their discrete force elements. This synchronicity is proposed as the mechanism for the strong force and for coherent assemblies. The same mechanism, acting through coherent assemblies of electrons, explains molecular bonding. Thus the Cordus theory provides force unification by providing a model for electro-magneto-gravitational-synchronous (EMGS) interactions as consequences of lineal, bending, torsion, and synchronicity effects respectively. The discrete force element is a 3-D composite structure, with a hand defined by the energisation sequence between the axes. This hand provides the matter/anti-matter species differentiation, which are denoted dexter and sinister respectively. We acknowledge that we have not described what these discrete forces and flux tubes comprise. Instead, the design method used to develop the Cordus theory simply shows that having such elements is a logical necessity for this solution.

Note 3. Energy sharing: The Cordus theory requires that assemblies of multiple particules bonded by the synchronous interaction necessarily adopt a common energy state. This is because the interconnectedness of the two particules via the synchronised discrete forces causes the frequencies also to synchronise, and hence via Planck's relation E $=\mathrm{hf}$ the two energy systems are the same. Furthermore the instantaneous communication 
provided by the fibril ensures that any energy disturbances or excesses are instantaneously (or at least at the next frequency cycle) communicated throughout the assembly.

Note 4. Alternative design: If we had taken a cis-phasic model at synchronisation (stage 2) then the photons would be sequential, and the original fibrils would need to persist for one half-frequency cycle longer, re-energising the other pair of reactive ends, collapsing their hyff, and creating a second photon out of the hyff. However this is not the preferred model here, though we mention it as it the evidence for its exclusion is not overwhelming.

\section{Copyrights}

Copyright for this article is retained by the author(s), with first publication rights granted to the journal.

This is an open-access article distributed under the terms and conditions of the Creative Commons Attribution license (http://creativecommons.org/licenses/by/3.0/). 